\title{
RADICALS AND IDEALS OF AFFINE NEAR-SEMIRINGS OVER BRANDT SEMIGROUPS
}

\author{
JITENDER KUMAR AND K. V. KRISHNA
}

\begin{abstract}
This work obtains all the right ideals, radicals, congruences and ideals of the affine near-semirings over Brandt semigroups.
\end{abstract}

\section{INTRODUCTION}

An algebraic structure $(\mathcal{N},+, \cdot)$ with two binary operations + and $\cdot$ is said to be a near-semiring if $(\mathcal{N},+)$ and $(\mathcal{N}, \cdot)$ are semigroups and $\cdot$ is one-side, say left, distributive over + , i.e. $a \cdot(b+c)=a \cdot b+a \cdot c$, for all $a, b, c \in \mathcal{N}$. Typical examples of near-semirings are of the form $M(S)$, the set of all mappings on a semigroup $S$. Near-semirings are not only the natural generalization of semirings and near-rings, but also they have very prominent applications in computer science. To name a few: process algebras by Bergstra and Klop [1, and domain axioms in near-semirings by Struth and Desharnais [3].

Near-semirings were introduced by van Hoorn and van Rootselaar as a generalization of near-rings [11. In [10, van Hoorn generalized the concept of Jacobson radical of rings to zero-symmetric near-semirings. These radicals also generalize the radicals of near-rings by Betsch [2]. In this context, van Hoorn introduced fourteen radicals of zero-symmetric near-semiring and studied some relation between them. The properties of these radicals are further investigated in the literature (e.g. 5, 12). Krishna and Chatterjee developed a radical (which is similar to the Jacobson radical of rings) for a special class of near-semirings to test the minimality of linear sequential machines in 6 .

In this paper, we study the ideals and radicals of the zero-symmetric affine near-semiring over a Brandt semigroup. First we present the necessary background material in Section 2. For the near-semiring under consideration, we obtain the right ideals in Section 3 and ascertain all radicals in Section 4. Further, we determine all its congruences and consequently obtain its ideals in Section 5 .

\section{Preliminaries}

In this section, we provide a necessary background material through two subsections. One is to present the notions of near-semirings, and their ideals and radicals. In the second subsection, we recall the notion of the affine near-semiring over a Brandt semigroup. We also utilize this section to fix our notations which used throughout the work.

2000 Mathematics Subject Classification. Primary 16Y99; Secondary 16N80, $20 \mathrm{M} 11$.

Key words and phrases. Near-semirings, Ideals, Radicals. 
2.1. A near-semiring and its radicals. In this subsection, we recall some necessary fundamentals of near-semirings from [5, 10, 11].

Definition 2.1. An algebraic structure $(\mathcal{N},+, \cdot)$ is said to be a near-semiring if

(1) $(\mathcal{N},+)$ is a semigroup,

(2) $(\mathcal{N}, \cdot)$ is a semigroup, and

(3) $a \cdot(b+c)=a \cdot b+a \cdot c$, for all $a, b, c \in \mathcal{N}$.

Furthermore, if there is an element $0 \in \mathcal{N}$ such that

(4) $a+0=0+a=a$ for all $a \in \mathcal{N}$, and

(5) $a \cdot 0=0 \cdot a=0$ for all $a \in \mathcal{N}$,

then $(\mathcal{N},+, \cdot)$ is called a zero-symmetric near-semiring.

Example 2.2. Let $(S,+)$ be a semigroup and $M(S)$ be the set of all functions on $S$. The algebraic structure $(M(S),+, \circ)$ is a near-semiring, where + is point-wise addition and $\circ$ is composition of mappings, i.e., for $x \in S$ and $f, g \in M(S)$,

$$
x(f+g)=x f+x g \quad \text { and } \quad x(f \circ g)=(x f) g .
$$

We write an argument of a function on its left, e.g. $x f$ is the value of a function $f$ at an argument $x$. We always denote the composition $f \circ g$ by $f g$. The notions of homomorphism and subnear-semiring of a near-semiring can be defined in a routine way.

Definition 2.3. Let $\mathcal{N}$ be a zero-symmetric near-semiring. A semigroup $(S,+)$ with identity $0_{S}$ is said to be an $\mathcal{N}$-semigroup if there exists a composition

$$
(s, a) \mapsto s a: S \times \mathcal{N} \longrightarrow S
$$

such that, for all $a, b \in \mathcal{N}$ and $s \in S$,

(1) $s(a+b)=s a+s b$,

(2) $s(a b)=(s a) b$, and

(3) $s 0=0_{S}$.

Note that the semigroup $(\mathcal{N},+)$ of a near-semiring $(\mathcal{N},+, \cdot)$ is an $\mathcal{N}$-semigroup. We denote this $\mathcal{N}$-semigroup by $\mathcal{N}^{+}$.

Definition 2.4. Let $S$ be an $\mathcal{N}$-semigroup. A semigroup congruence $\sim_{r}$ of $S$ is said to be a congruence of $\mathcal{N}$-semigroup $S$, if for all $s, t \in S$ and $a \in \mathcal{N}$,

$$
s \sim_{r} t \Longrightarrow s a \sim_{r} t a .
$$

Definition 2.5. An $\mathcal{N}$-morphism of an $\mathcal{N}$-semigroup $S$ is a semigroup homomorphism $\phi$ of $S$ into an $\mathcal{N}$-semigroup $S^{\prime}$ such that

$$
(s a) \phi=(s \phi) a
$$

for all $a \in \mathcal{N}$ and $s \in S$. The kernel of an $\mathcal{N}$-morphism is called an $\mathcal{N}$-kernel of an $\mathcal{N}$-semigroup $S$. A subsemigroup $T$ of an $\mathcal{N}$-semigroup $S$ is said to be $\mathcal{N}$ subsemigroup of $S$ if and only if $0_{S} \in T$ and $T \mathcal{N} \subseteq T$.

Definition 2.6. The kernel of a homomorphism of $\mathcal{N}$ is called an ideal of $\mathcal{N}$. The $\mathcal{N}$-kernels of the $\mathcal{N}$-semigroup $\mathcal{N}^{+}$are called right ideals of $\mathcal{N}$.

One may refer to [10, 11] for a few other notions viz. strong ideal, modular right ideal and $\lambda$-modular right ideal, a special congruence $r_{\Delta}^{\prime \prime}$ associated to a normal subsemigroup $\Delta$ of a semigroup $S$, and, for various $(\nu, \mu)$, the $\mathcal{N}$-semigroups of type $(\nu, \mu)$. The homomorphism corresponding to $r_{\Delta}^{\prime \prime}$ is denoted by $\lambda_{\Delta}$. 


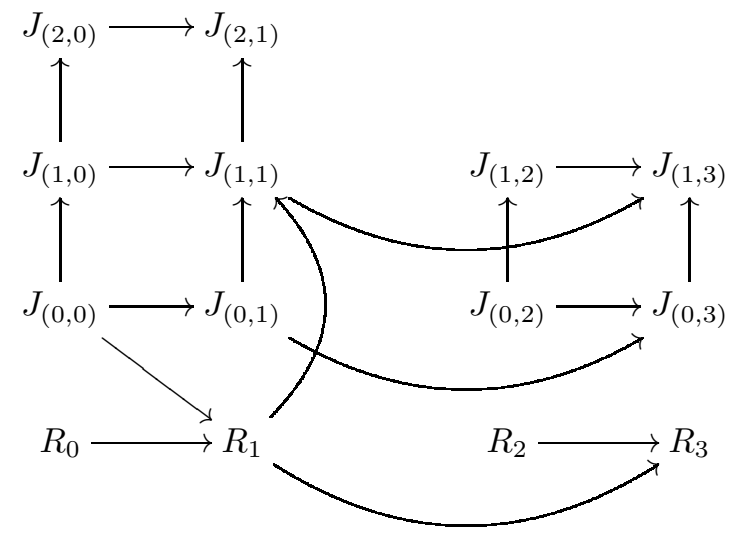

FIGURE 1. Relation between various radicals of a near-semiring

Definition 2.7. Let $s$ be an element of an $\mathcal{N}$-semigroup $S$. The annihilator of $s$, denoted by $A(s)$, defined by the set $\left\{a \in \mathcal{N}: s a=0_{S}\right\}$. Further, for a subset $T$ of $S$, the annihilator of $T$ is

$$
A(T)=\bigcap_{s \in T} A(s)=\left\{a \in \mathcal{N}: T a=0_{S}\right\} .
$$

Theorem 2.8 ([5]). The annihilator $A(S)$ of an $\mathcal{N}$-semigroup $S$ is an ideal of $\mathcal{N}$.

We now recall the notions of various radicals in the following definition.

Definition 2.9 ([10]). Let $\mathcal{N}$ be a zero-symmetric near-semiring.

(1) For $\nu=0,1$ with $\mu=0,1,2,3$ and $\nu=2$ with $\mu=0,1$

$$
J_{(\nu, \mu)}(\mathcal{N})=\bigcap_{S \text { is of type }(\nu, \mu)} A(S) .
$$

(2) $R_{0}(\mathcal{N})$ is the intersection of all maximal modular right ideals of $\mathcal{N}$.

(3) $R_{1}(\mathcal{N})$ is the intersection of all modular maximal right ideals of $\mathcal{N}$.

(4) $R_{2}(\mathcal{N})$ is the intersection of all maximal $\lambda$-modular right ideals of $\mathcal{N}$.

(5) $R_{3}(\mathcal{N})$ is the intersection of all $\lambda$-modular maximal right ideals of $\mathcal{N}$.

In any case, the empty intersection of subsets of $\mathcal{N}$ is $\mathcal{N}$. The relations between these radicals are given in Figure 1, where $A \rightarrow B$ means $A \subset B$.

Remark 2.10 (2, 4, 9]). If $\mathcal{N}$ is a near-ring, then $J_{(0, \mu)}(\mathcal{N}), \mu=0,1,2,3$ are the radical $J_{0}(\mathcal{N}) ; J_{(1, \mu)}(\mathcal{N}), \mu=0,1,2,3$ are the radical $J_{1}(\mathcal{N}) ; J_{(2, \mu)}(\mathcal{N}), \mu=0,1$, are the radical $J_{2}(\mathcal{N})$; and $R_{\nu}(\mathcal{N}), \nu=0,1,2,3$ are the radical $D(\mathcal{N})$ of Betsch. Further, if $\mathcal{N}$ is a ring, then all the fourteen radicals are the radical of Jacobson.

Definition 2.11. A zero-symmetric near-semiring $\mathcal{N}$ is called $(\nu, \mu)$-primitive if $\mathcal{N}$ has an $\mathcal{N}$-semigroup $S$ of type $(\nu, \mu)$ with $A(S)=\{0\}$. 
2.2. An affine near-semiring over a Brandt semigroup. In this subsection, we present the necessary fundamentals of affine near-semirings over Brandt semigroups. For more detail one may refer to [7, 8].

Let $(S,+)$ be a semigroup. An element $f \in M(S)$ is said to be an affine map if $f=g+h$, for some endomorphism $g$ and a constant map $h$ on $S$. The set of all affine mappings over $S$, denoted by $\operatorname{Aff}(S)$, need not be a subnear-semiring of $M(S)$. The affine near-semiring, denoted by $A^{+}(S)$, is the subnear-semiring generated by $\operatorname{Aff}(S)$ in $M(S)$. Indeed, the subsemigroup of $(M(S),+)$ generated by $\operatorname{Aff}(S)$ equals $\left(A^{+}(S),+\right)$ (cf. [6. Corollary 1]). If $(S,+)$ is commutative, then $\operatorname{Aff}(S)$ is a subnear-semiring of $M(S)$ so that $\operatorname{Aff}(S)=A^{+}(S)$.

Definition 2.12. For any integer $n \geq 1$, let $[n]=\{1,2, \ldots, n\}$. The semigroup $\left(B_{n},+\right)$, where $B_{n}=([n] \times[n]) \cup\{\vartheta\}$ and the operation + is given by

$$
(i, j)+(k, l)=\left\{\begin{array}{cl}
(i, l) & \text { if } j=k ; \\
\vartheta & \text { if } j \neq k
\end{array}\right.
$$

and, for all $\alpha \in B_{n}, \alpha+\vartheta=\vartheta+\alpha=\vartheta$, is known as Brandt semigroup. Note that $\vartheta$ is the (two sided) zero element in $B_{n}$.

Let $\vartheta$ be the zero element of the semigroup $(S,+)$. For $f \in M(S)$, the support of $f$, denoted by $\operatorname{supp}(f)$, is defined by the set

$$
\operatorname{supp}(f)=\{\alpha \in S \mid \alpha f \neq \vartheta\} .
$$

A function $f \in M(S)$ is said to be of $k$-support if the cardinality of $\operatorname{supp}(f)$ is $k$, i.e. $|\operatorname{supp}(f)|=k$. If $k=|S|$ (or $k=1$ ), then $f$ is said to be of full support (or singleton support, respectively). For $X \subseteq M(S)$, we write $X_{k}$ to denote the set of all mappings of $k$-support in $X$, i.e.

$$
X_{k}=\{f \in X \mid f \text { is of } k \text {-support }\} .
$$

For ease of reference, we continue to use the following notations for the elements of $M\left(B_{n}\right)$, as given in [8].

\section{Notation 2.13.}

(1) For $c \in B_{n}$, the constant map that sends all the elements of $B_{n}$ to $c$ is denoted by $\xi_{c}$. The set of all constant maps over $B_{n}$ is denoted by $\mathcal{C}_{B_{n}}$.

(2) For $k, l, p, q \in[n]$, the singleton support map that send $(k, l)$ to $(p, q)$ is denoted by ${ }^{(k, l)} \zeta_{(p, q)}$.

(3) For $p, q \in[n]$, the $n$-support map which sends $(i, p)$ (where $1 \leq i \leq n$ ) to $(i \sigma, q)$ using a permutation $\sigma \in S_{n}$ is denoted by $(p, q ; \sigma)$. We denote the identity permutation on $[n]$ by $i d$.

Note that $A^{+}\left(B_{1}\right)=\{(1,1 ; i d)\} \cup \mathcal{C}_{B_{1}}$. For $n \geq 2$, the elements of $A^{+}\left(B_{n}\right)$ are given by the following theorem.

Theorem $2.14([8])$. For $n \geq 2, A^{+}\left(B_{n}\right)$ precisely contains $(n !+1) n^{2}+n^{4}+1$ elements with the following breakup.

(1) All the $n^{2}+1$ constant maps.

(2) All the $n^{4}$ singleton support maps.

(3) The remaining $(n !) n^{2}$ elements are the $n$-support maps of the form $(p, q ; \sigma)$, where $p, q \in[n]$ and $\sigma \in S_{n}$. 
We are ready to investigate the radicals and ideals of $A^{+}\left(B_{n}\right)$ - the affine nearsemiring over a Brandt semigroup. Since the radicals are defined in the context of zero-symmetric near-semirings, we extend the semigroup reduct $\left(A^{+}\left(B_{n}\right),+\right)$ to monoid by adjoining 0 and make the resultant near-semiring zero-symmetric. In what follows, the zero-symmetric affine near-semiring $A^{+}\left(B_{n}\right) \cup\{0\}$ is denoted by $\mathcal{N}$, i.e.

(1) $(\mathcal{N},+)$ is a monoid with identity element 0 ,

(2) $(\mathcal{N}, \circ)$ is a semigroup,

(3) $0 f=f 0=0$, for all $f \in \mathcal{N}$, and

(4) $f(g+h)=f g+f h$, for all $f, g, h \in \mathcal{N}$.

In this work, a nontrivial congruence of an algebraic structure is meant to be a congruence which is neither equality nor universal relation.

\section{Right IDEALS}

In this section, we obtain all the right ideals of the affine near-semiring $\mathcal{N}$ by ascertaining the concerning congruences of $\mathcal{N}$-semigroups. We begin with the following lemma.

Lemma 3.1. Let $\sim$ be a nontrivial congruence over the semigroup $(\mathcal{N},+)$ and $f \in A^{+}\left(B_{n}\right)_{n^{2}+1}$. If $f \sim \xi_{\vartheta}$, then $\sim=\left(A^{+}\left(B_{n}\right) \times A^{+}\left(B_{n}\right)\right) \cup\{(0,0)\}$.

Proof. First note that $\left(A^{+}\left(B_{n}\right) \times A^{+}\left(B_{n}\right)\right) \cup\{(0,0)\}$ is a congruence relation of the semigroup $(\mathcal{N},+)$. Let $f=\xi_{\left(p_{0}, q_{0}\right)}$ and $\xi_{(p, q)}$ be an arbitrary full support map. Since

$$
\xi_{(p, q)}=\xi_{\left(p, p_{0}\right)}+\xi_{\left(p_{0}, q_{0}\right)}+\xi_{\left(q_{0}, q\right)} \sim \xi_{\left(p, p_{0}\right)}+\xi_{\vartheta}+\xi_{\left(q_{0}, q\right)}=\xi_{\vartheta}
$$

we have $\xi_{(p, q)} \sim \xi_{\vartheta}$ for all $p, q \in[n]$. Further, given an arbitrary $n$-support map $(k, l ; \sigma)$, since $\xi_{(p, l)} \sim \xi_{\vartheta}$, we have

$$
(k, l ; \sigma)=(k, p ; \sigma)+\xi_{(p, l)} \sim(k, p ; \sigma)+\xi_{\vartheta}=\xi_{\vartheta} .
$$

Thus, all $n$-support maps are related to $\xi_{\vartheta}$ under $\sim$. Similarly, given an arbitrary ${ }^{(k, l)} \zeta_{(p, q)} \in A^{+}\left(B_{n}\right)_{1}$, since $\xi_{(p, q)} \sim \xi_{\vartheta}$, for $\sigma \in S_{n}$ such that $k \sigma=q$, we have

$$
{ }^{(k, l)} \zeta_{(p, q)}=\xi_{(p, q)}+(l, q ; \sigma) \sim \xi_{\vartheta}+(l, q ; \sigma)=\xi_{\vartheta} .
$$

Hence, all elements of $A^{+}\left(B_{n}\right)$ are related to each other under $\sim$.

Now, using Lemma 3.1, we determine the right ideals of $\mathcal{N}$ in the following theorem.

Theorem 3.2. $\mathcal{N}$ and $\{0\}$ are only the right ideals of $\mathcal{N}$.

Proof. Let $I \neq\{0\}$ be a right ideal of $\mathcal{N}$ so that $I=\operatorname{ker} \varphi$, where $\varphi: \mathcal{N}^{+} \longrightarrow S$ is an $\mathcal{N}$-morphism. Note that $I=[0]_{\sim_{r}}$, where $\sim_{r}$ is the congruence over the $\mathcal{N}$-semigroup $\mathcal{N}^{+}$defined by $a \sim_{r} b$ if and only if $a \varphi=b \varphi$, i.e. the relation $\sim_{r}$ on $\mathcal{N}$ is compatible with respect to + and if $a \sim_{r} b$ then $a c \sim_{r} b c$ for all $c \in \mathcal{N}$.

Let $f$ be a nonzero element of $\mathcal{N}$ such that $f \sim_{r} 0$. First note that

$$
\xi_{\vartheta}=f \xi_{\vartheta} \sim_{r} 0 \xi_{\vartheta}=0
$$

Further, for any full support map $\xi_{(p, q)}$, we have

$$
\xi_{(p, q)}=f \xi_{(p, q)} \sim_{r} 0 \xi_{(p, q)}=0
$$

so that, by transitivity, $\xi_{(p, q)} \sim_{r} \xi_{\vartheta}$. Hence, by Lemma 3.1. $\sim_{r}=\mathcal{N} \times \mathcal{N}$ so that $I=\mathcal{N}$. 
Remark 3.3. The ideal $\{0\}$ is the maximal right ideal of $\mathcal{N}$.

\section{RADICALS}

In order to obtain the radicals of the affine near-semiring $\mathcal{N}$, in this section, we first identify an $\mathcal{N}$-semigroup which satisfies the criteria of all types of $\mathcal{N}$ semigroups by van Hoorn. Using the $\mathcal{N}$-semigroup, we ascertain the radicals of $\mathcal{N}$. Further, we observe that the near-semiring $\mathcal{N}$ is $(\nu, \mu)$-primitive (cf. Theorem 4.3).

Consider the subsemigroup $\mathcal{C}=\mathcal{C}_{B_{n}} \cup\{0\}$ of $(\mathcal{N},+)$ and observe that $\mathcal{C}$ is an $\mathcal{N}$-semigroup with respect to the multiplication in $\mathcal{N}$. The following properties of the $\mathcal{N}$-semigroup $\mathcal{C}$ are useful.

\section{Lemma 4.1.}

(1) Every nonzero element of $\mathcal{C}$ is a generator. Moreover, the $\mathcal{N}$-semigroup $\mathcal{C}$ is strongly monogenic and $A(g)=\{0\}$ for all $g \in \mathcal{C} \backslash\{0\}$.

(2) The subsemigroup $\{0\}$ is the maximal $\mathcal{N}$-subsemigroup of $\mathcal{C}$.

(3) The $\mathcal{N}$-semigroup $\mathcal{C}$ is irreducible.

Proof.

(1) Let $g \in \mathcal{C}_{B_{n}}$. Note that $g \mathcal{N} \subseteq \mathcal{C}$ because the product of a constant map with any map is a constant map. Conversely, for $f \in \mathcal{C}$, since $g f=f$, we have $g \mathcal{N}=\mathcal{C}$ for all $g \in \mathcal{C} \backslash\{0\}$. Further, since $0 \mathcal{N}=\{0\}$ and $\mathcal{C N}=\mathcal{C} \neq\{0\}$. Hence, $\mathcal{C}$ is strongly monogenic.

(2) We show that the semigroups $\mathcal{C}$ and $\{0\}$ are the only $\mathcal{N}$-subsemigroups of $\mathcal{C}$. Let $T$ be an $\mathcal{N}$-subsemigroup of $\mathcal{C}$ such that $\{0\} \neq T \subsetneq \mathcal{C}$. Then there exist $f(\neq 0) \in T$ and $g \in \mathcal{C} \backslash T$. Since $f g=g \notin T$, we have $T \mathcal{N} \nsubseteq T$; a contradiction to $T$ is an $\mathcal{N}$-subsemigroup. Hence, the result.

(3) By Lemma 4.1(1), the $\mathcal{N}$-semigroup $\mathcal{C}$ is monogenic with any nonzero element $g$ as generator such that $A(g)=\{0\}$; thus, $A(g)$ is maximal right ideal in $\mathcal{N}$ (cf. Remark [3.3). Hence, by [10, Theorem 8], $\mathcal{C}$ is irreducible.

Remark 4.2. Since a strongly monogenic $\mathcal{N}$-semigroup is monogenic we have, for $\mu=0,1,2,3$, an $\mathcal{N}$-semigroup of type $(1, \mu)$ is of type $(0, \mu)$.

Theorem 4.3. For $\nu=0,1$ with $\mu=0,1,2,3$ and $\nu=2$ with $\mu=0,1$, we have the following.

(1) The $\mathcal{N}$-semigroup $\mathcal{C}$ is of type $(\nu, \mu)$ with $A(\mathcal{C})=0$.

(2) The near-semiring $\mathcal{N}$ is $(\nu, \mu)$-primitive for all $\nu$ and $\mu$.

(3) $J_{(\nu, \mu)}(\mathcal{N})=\{0\}$ for all $\nu$ and $\mu$.

Proof. In view of Remark 4.2, we prove (1) in the following cases.

Type $(1, \mu)$ : Note that, by Lemma $4.1(1)$, the $\mathcal{N}$-semigroup $\mathcal{C}$ is strongly monogenic.

(i) By Lemma 4.1(3), we have $\mathcal{C}$ is irreducible. Hence, $\mathcal{C}$ is of type $(1,0)$.

(ii) By Lemma 4.1(1) and Remark 3.3, for any generator $g, A(g)$ is a maximal right ideal. Hence, $\mathcal{C}$ is of type $(1,1)$.

(iii) Note that the ideal $\{0\}$ is strong right ideal so that for any generator $g, A(g)$ is a strong maximal right ideal (see ii above). Further, note that $A(g)$ is a maximal strong right ideal (cf. Remark 3.3). Hence, $\mathcal{C}$ is of type $(1,2)$ and $(1,3)$. 
Type $(2, \mu)$ : Since $\mathcal{C}$ is monogenic and, for any generator $g$ of $\mathcal{C}, A(g)$ is a maximal $\mathcal{N}$-subsemigroup of $\mathcal{C}$ (cf. Lemma 4.1(1) and Lemma 4.1(2)). Thus, $\mathcal{C}$ is of type $(2,1)$. By [10, Theorem 9], every $\mathcal{N}$-semigroup of type $(2,1)$ will be of type $(2,0)$. Hence, $\mathcal{C}$ is of type $(2,0)$.

Proofs for (2) and (3) follow from (1).

Theorem 4.4. For $\nu=0,1$, we have $R_{\nu}(\mathcal{N})=\{0\}$.

Proof. In view of Figure 1, we prove that result by showing that the right ideal $\{0\}$ is a modular maximal right ideal. By Lemma 4.1(1), the $\mathcal{N}$-semigroup $\mathcal{C}$ is monogenic and has a generator $g$ such that $A(g)=\{0\}$. Hence, the right ideal $\{0\}$ is modular (cf. [10, Theorem 7]). Further, since $\{0\}$ is a maximal right ideal (cf. Remark 3.3), we have $\{0\}$ is a modular maximal right ideal.

Theorem 4.5. For $\nu=2,3$, we have $R_{\nu}(\mathcal{N})=\mathcal{N}$.

Proof. In view of Figure 1 and Theorem 3.2 we prove that the homomorphism $\lambda_{\{0\}}$ is not modular. Note that the congruence relation $r_{\{0\}}^{\prime \prime}$ is the equality relation on $(\mathcal{N},+)$, where $r_{\{0\}}^{\prime \prime}$ is the transitive closure of the two sided stable reflexive and symmetric relation relation $r_{\{0\}}$ associated with a normal subsemigroup $\{0\}$ of the semigroup $(\mathcal{N},+)$. Consequently, the semigroup homomorphism $\lambda_{\{0\}}$ is an identity map on $(\mathcal{N},+)$. If the morphism $\lambda_{\{0\}}$ is modular, then there is an element $u \in \mathcal{N}$ such that $x=u x$ for all $x \in \mathcal{N}$, but there is no left identity element in $\mathcal{N}$. Consequently, $\lambda_{\{0\}}$ is not modular. Thus, there is no maximal $\lambda$-modular right ideal. Hence, for $\nu=2,3$, we have $R_{\nu}(\mathcal{N})=\mathcal{N}$.

\section{IDEALS}

In this section, we prove that there is only one nontrivial congruence relation on $\mathcal{N}$ (cf. Theorem 5.1). Consequently, all the ideals of $\mathcal{N}$ are determined.

Theorem 5.1. The near-semiring $\mathcal{N}$ has precisely the following congruences.

(1) Equality relation

(2) $\mathcal{N} \times \mathcal{N}$

(3) $\left(A^{+}\left(B_{n}\right) \times A^{+}\left(B_{n}\right)\right) \cup\{(0,0)\}$

Hence, $\mathcal{N}$ and $\{0\}$ are the only ideals of the near-semiring $\mathcal{N}$.

Proof. In the sequel, we prove the theorem through the following claims.

Claim 1: Let $\sim$ be a nontrivial congruence over the near-semiring $\mathcal{N}$ and $f \in$ $\mathcal{N} \backslash\left\{0, \xi_{\vartheta}\right\}$. If $f \sim \xi_{\vartheta}$, then $\sim=\left(A^{+}\left(B_{n}\right) \times A^{+}\left(B_{n}\right)\right) \cup\{(0,0)\}$.

Proof: First note that $\left(A^{+}\left(B_{n}\right) \times A^{+}\left(B_{n}\right)\right) \cup\{(0,0)\}$ is a congruence relation of the near-semiring $\mathcal{N}$. If $f \in A^{+}\left(B_{n}\right)_{n^{2}+1}$, since $\sim$ is a congruence of the semigroup $(\mathcal{N},+)$, by Lemma 3.1 we have the result. Otherwise, we reduce the problem to Lemma 3.1 in the following cases.

Case 1.1: $f$ is of singleton support. Let $f={ }^{(k, l)} \zeta_{(p, q)}$. Since ${ }^{(k, l)} \zeta_{(p, q)} \sim \xi_{\vartheta}$ we have

$$
\xi_{(k, l)}{ }^{(k, l)} \zeta_{(p, q)} \sim \xi_{(k, l)} \xi_{\vartheta}
$$

so that $\xi_{(p, q)} \sim \xi_{\vartheta}$.

Case 1.2: $f$ is of $n$-support. Let $f=(p, q ; \sigma)$. Since $(p, q ; \sigma) \sim \xi_{\vartheta}$ we have

$$
\xi_{(k, p)}(p, q ; \sigma) \sim \xi_{(k, p)} \xi_{\vartheta}
$$

so that $\xi_{(k \sigma, q)} \sim \xi_{\vartheta}$. 
Claim 2: If two nonzero elements are in one class under a nontrivial congruence over $\mathcal{N}$, then the congruence is $\left(A^{+}\left(B_{n}\right) \times A^{+}\left(B_{n}\right)\right) \cup\{(0,0)\}$.

Proof: Let $f, g \in \mathcal{N} \backslash\{0\}$ such that $f \sim g$ under a congruence $\sim$ over $\mathcal{N}$. If $f$ or $g$ is equal to $\xi_{\vartheta}$, then by Claim 1, we have the result. Otherwise, we consider the following six cases classified by the supports of $f$ and $g$. In each case, we show that there is an element $h \in A^{+}\left(B_{n}\right) \backslash\left\{\xi_{\vartheta}\right\}$ such that $h \sim \xi_{\vartheta}$ so that the result follows from Claim 1.

Case 2.1: $f, g \in A^{+}\left(B_{n}\right)_{1}$. Let $f={ }^{(i, j)} \zeta_{(k, l)}$ and $g={ }^{(s, t)} \zeta_{(u, v)}$. If $(i, j) \neq(s, t)$, we have

$$
\xi_{\vartheta}={ }^{(i, j)} \zeta_{(k, l)}+{ }^{(s, t)} \zeta_{(v, v)} \sim{ }^{(s, t)} \zeta_{(u, v)}+{ }^{(s, t)} \zeta_{(v, v)}={ }^{(s, t)} \zeta_{(u, v)} .
$$
have

Otherwise, $(i, j)=(s, t)$ so that $(k, l) \neq(u, v)$. Now, if $k \neq u$, then we

$$
{ }^{(i, j)} \zeta_{(k, l)}={ }^{(i, j)} \zeta_{(k, k)}+{ }^{(i, j)} \zeta_{(k, l)} \sim{ }^{(i, j)} \zeta_{(k, k)}+{ }^{(i, j)} \zeta_{(u, v)}=\xi_{\vartheta} .
$$

Similarly, if $l \neq v$, we have

$$
\xi_{\vartheta}={ }^{(i, j)} \zeta_{(k, l)}+{ }^{(i, j)} \zeta_{(v, v)} \sim{ }^{(i, j)} \zeta_{(u, v)}+{ }^{(i, j)} \zeta_{(v, v)}={ }^{(i, j)} \zeta_{(u, v)} .
$$

Case 2.2: $f, g \in A^{+}\left(B_{n}\right)_{n^{2}+1}$. Let $f=\xi_{(k, l)}$ and $g=\xi_{(u, v)}$. By considering full support maps whose images are the same as in various subcases of Case 1 , we can show that there is an element in $A^{+}\left(B_{n}\right) \backslash\left\{\xi_{\vartheta}\right\}$ that is related to $\xi_{\vartheta}$ under $\sim$.

Case 2.3: $f, g \in A^{+}\left(B_{n}\right)_{n}$. Let $f=(i, j ; \sigma)$ and $g=(k, l ; \rho)$. If $l \neq j$, then

$$
(i, j ; \sigma)=(i, j ; \sigma)+\xi_{(j, j)} \sim(k, l ; \rho)+\xi_{(j, j)}=\xi_{\vartheta} .
$$

Otherwise, we have $(i, j ; \sigma) \sim(k, j ; \rho)$. Now, if $i \neq k$, then

$$
\xi_{\vartheta}=(k, k ; i d)(i, j ; \sigma) \sim(k, k ; i d)(k, j ; \rho)=(k, j ; \rho) .
$$

In case $i=k$, we have $\sigma \neq \rho$. Thus, there exists $t \in[n]$ such that $t \sigma \neq t \rho$. Now, $(i, j ; \sigma) \sim(i, j ; \rho)$ implies $\xi_{(k, i)}(i, j ; \sigma) \sim \xi_{(k, i)}(i, j ; \rho)$, i.e. $\xi_{(k \sigma, j)} \sim$ $\xi_{(k \rho, j)}$. Consequently,

$$
\xi_{(k \sigma, j)}=\xi_{(k \sigma, k \sigma)}+\xi_{(k \sigma, j)} \sim \xi_{(k \sigma, k \sigma)}+\xi_{(k \rho, j)}=\xi_{\vartheta} .
$$

Case 2.4: $f \in A^{+}\left(B_{n}\right)_{1}, g \in A^{+}\left(B_{n}\right)_{n^{2}+1}$. Let $f={ }^{(k, l)} \zeta_{(p, q)}$ and $g=\xi_{(i, j)}$. Now, for $(s, t) \neq(k, l)$, we have

$$
\xi_{\vartheta}=\xi_{(s, t)} f \sim \xi_{(s, t)} g=\xi_{(i, j)} .
$$

Case 2.5: $f \in A^{+}\left(B_{n}\right)_{n^{2}+1}, g \in A^{+}\left(B_{n}\right)_{n}$. Let $f=\xi_{(p, q)}$ and $g=(i, j ; \sigma)$. Now, for $l \neq i$, we have

$$
{ }^{(k, l)} \zeta_{(p, q)}={ }^{(k, l)} \zeta_{(p, p)}+f \sim{ }^{(k, l)} \zeta_{(p, p)}+g=\xi_{\vartheta} .
$$

Case 2.6: $f \in A^{+}\left(B_{n}\right)_{1}, g \in A^{+}\left(B_{n}\right)_{n}$. Let $f={ }^{(k, l)} \zeta_{(p, q)}$ and $g=(i, j ; \sigma)$. Now, for $l \neq i$, we have

$$
\xi_{\vartheta}=\xi_{(i, i)} f \sim \xi_{(i, i)} g=\xi_{(i \sigma, j)} .
$$




\section{REFERENCES}

[1] J. A. Bergstra and J. W. Klop. An Introduction to Process Algebra, volume 17 of Cambridge Tracts Theoret. Comput. Sci. Cambridge Univ. Press, Cambridge, 1990.

[2] G. Betsch. Struktursätze für Fastringe. Inaugural-Dissertation. Eberhard-Karls-Universität zu Tübingen. 1963.

[3] J. Desharnais and G. Struth. Domain axioms for a family of near-semirings. In $A M A S T$, pages 330-345, 2008.

[4] N. Jacobson. Structure of rings. American Mathematical Society Colloquium Publications, Vol. 37. Revised edition. American Mathematical Society, Providence, R.I., 1964.

[5] K. V. Krishna. Near-Semirings: Theory and Application. PhD thesis, IIT Delhi, New Delhi, 2005.

[6] K. V. Krishna and N. Chatterjee. A necessary condition to test the minimality of generalized linear sequential machines using the theory of near-semirings. Algebra Discrete Math., (3):3045,2005

[7] J. Kumar. Affine Near-Semirings over Brandt Semigroups. PhD thesis, IIT Guwahati, 2014.

[8] J. Kumar and K. V. Krishna. Affine near-semirings over Brandt semigroups. Comm. Algebra, 42(12):5152-5169, 2014.

[9] G. Pilz. Near-Rings: The Theory and Its Applications, volume 23 of North-Holland Mathematics Studies. North-Holland Publishing Company, 1983.

[10] W. G. van Hoorn. Some generalisations of the Jacobson radical for semi-nearrings and semirings. Math. Z., 118:69-82, 1970.

[11] W. G. van Hoorn and B. van Rootselaar. Fundamental notions in the theory of seminearrings. Compositio Math., 18:65-78, 1967.

[12] M. Zulfiqar. A note on radicals of seminear-rings. Novi Sad J. Math., 39(1):65-68, 2009.

Department of Mathematics, Indian Institute of Technology Guwahati, Guwahati, INDIA

E-mail address: $\{$ jitender, kvk\}@iitg.ac.in 